\title{
The utility of the preoperative neutrophil-to- lymphocyte ratio in predicting severe cholecystitis: a retrospective cohort study
}

\author{
Sang Kuon Lee, Sang Chul Lee, Jae Woo Park and Say-June Kim*
}

\begin{abstract}
Background: To evaluate whether the neutrophil-to-lymphocyte ratio (NLR), as a prognostic indicator, in patients can differentiate between simple and severe cholecystitis.

Methods: A database of 632 patients who underwent cholecystectomy due to cholecystitis during approximately a seven-year span in a single institution was evaluated. Severe cholecystitis was defined when the cholecystitis was complicated by secondary changes, including hemorrhage, gangrene, emphysema, and perforation. The NLR was calculated at admission as the absolute neutrophil count divided by the absolute lymphocyte count. We used receiver operating characteristic curve analysis to identify the optimal value for the NLR in relation to the severity of cholecystitis. Thereafter, the differences in clinical manifestations according to the NLR cut-off value were investigated.

Results: Our study population comprised 503 patients with simple cholecystitis (79.6\%) and 129 patients with severe cholecystitis (20.4\%). The NLR of 3.0 could predict severe cholecystitis with $70.5 \%$ sensitivity and $70.0 \%$ specificity. A higher NLR ( $\geq 3.0)$ was significantly associated with older age ( $p=0.001$ ), male gender ( $p=0.001)$, admission via the emergency department $(p<0.001)$, longer operation time $(p<0.001)$, higher incidence of postoperative complications ( $p=0.056)$, and prolonged length of hospital stay (LOS) $(p<0.001)$. Multivariate analysis found that patient age $\geq 50$ years (odds ratio [OR]: 2.312 , 95\% confidence interval [CI]: 1.472-3.630, $p<0.001$ ), preoperative NLR $\geq 3.0$ (OR: 1.876, $95 \%$ Cl: $1.246-2.825, p=0.003$ ), and admission via the emergency department (OR: 1.764, 95\% Cl: 1.170-2.660, $p=0.007$ ) were independent factors associated with prolonged LOS.
\end{abstract}

Conclusions: NLR $\geq 3.0$ was significantly associated with severe cholecystitis and prolonged LOS in patients undergoing cholecystectomy. Therefore, preoperative NLR in patients undergoing cholecystits due to cholecystitis seemed to be a useful surrogate marker for severe cholecystitis.

Keywords: Cholecystitis, Prognosis, Neutrophil-to-lymphocyte ratio, Length of hospital stay

\section{Background}

Acute cholecystitis accounts for most of the hospital admissions related to gastrointestinal diseases [1]. In approximately $90 \%$ of patients, inflammation develops due to obstruction of the cystic duct by one or more gallstones [2]. Delayed management can lead to increased morbidity, due to progression to severe cholecystitis, such as gangrenous change, abscess formation, and gallbladder perforation. The prevalence of severe cholecyst-

\footnotetext{
* Correspondence: sejoonkim@hanmail.net

Department of Surgery, Daejeon St. Mary's Hospital, College of Medicine, the Catholic University of Korea, Daeheung-dong 520-2, Joong-gu, Daejeon, Republic of Korea
}

itis has been reported to be $22-30 \%$ in surgical series $[3,4]$. Unfortunately, patients with severe cholecystitis are often challenging to accurately diagnose, both clinically and radiologically, since the clinical manifestations are unpredictable, and imaging studies are often equivocal [5]. However, marked contrasts in the morbidity and mortality rates have been observed beween patients with simple cholecystitis and severe cholecystitis $[3,6]$. Therefore, prompt detection and proper management of patients at risk of severe cholecystitis are essential in preventing associated complications.

To predict the prognosis of inflammatory diseases and some malignancies, several inflammation-based scores 
have been suggested, including the Modified Glasgow Prognostic Score, neutrophil-to-lymphocyte ratio (NLR), platelet-to-lymphocyte ratio, and prognostic Nutritional Index $[7,8]$. Of these, the NLR has received great interest, since it is simple to calculate, and involves no additional cost, as it uses results from a standard complete blood count test. The NLR is derived from the counts of circulating neutrophils and lymphocytes, both of which are major leukocyte subpopulations. The inflammation-triggered release of arachidonic acid metabolites and platelet-activating factors results in neutrophilia, and cortisol-induced stress results in relative lymphopenia, and thus, the NLR accurately represents the underlying inflammatory process [9]. Increasing evidence supports the utility of the NLR in predicting the prognosis of inflammatory and malignant diseases, although the application of the NLR to inflammatory gallbladder disease has not been reported. In the present study, we aimed to evaluate the utility of the NLR as a prognostic indicator in patients with cholecystitis, and to identify a relevant NLR value that discriminates between simple and severe cholecystitis.

\section{Methods}

\section{Study design and data collection}

We retrospectively reviewed prospectively collected data from patients who underwent cholecystectomies in Daejeon St. Mary's Hospital, the Catholic University of Korea, between March 2007 and February 2014. Furthermore, we verified the current data, and obtained additional data, by including the radiology and pathology reports as a part of this study. This study was approved by the ethics committee, Daejeon St. Mary's hospital, College of Medicine, the Catholic University of Korea (IRB code: DC13RISI0087).

During the study period, 1,023 cholecystectomies were performed either by the open or laparoscopic approach. To clearly identify gallbladder inflammation, we only selected 993 patients in whom the pathology reports indicated the presence of cholecystitis. Of these, we first excluded the patients $(\mathrm{n}=197)$ who initially presented with no specific symptom for cholecystitis. We then excluded patients $(\mathrm{n}=164)$ whose time-to-incision, the time interval between arrival to the hospital and the performance of surgical incision, was $120 \mathrm{~h}$ or longer. Finally, a toal of 632 patients were selected for inclusion in this study. Each patient's NLR was calculated at admission as the absolute neutrophil count divided by the absolute lymphocyte count, using data from their standard complete blood count test.

We conducted receiver operating characteristic (ROC) curve analyses to determine the cut-off value for preoperative NLR that could discriminate between simple and severe cholecystitis. The most prominent point on the ROC curve was chosen as the cut-off value for the
NLR. We then divided the patient population into two groups, according to the cut-off value of NLR, and attempted to detect differences in the clinical variables between these two groups. Thereafter, we performed univariate and multivariate analyses to investigate the effect of the NLR on the length of hospital stay (LOS).

\section{Terminology and definitions}

Cholecystitis was defined by a histological finding of an inflammatory infiltrate on examination of the gallbladder wall. Severe cholecystitis was defined as a cholecystitis complicated by secondary changes, including hemorrhage, gangrene, emphysema, or perforation, and/or when the pathological examination indicated xanthogranulomatous cholecystitis; the representative forms of severe cholecystitis were gangrenous cholecystitis and gallbladder perforation. All other pathological findings were categorized as simple cholecystitis. Conversion was defined as the completion of any part of a procedure using an open technique except for minimal wound extension $(\leq 10 \mathrm{~mm})$ for specimen delivery. In addition, the incidence of an addition of ports during surgery was also counted. Operation time referred to the time interval between the initial skin incision and completion of wound closure, as documented by an anesthesiologist. With regard to intraoperative complications, bleeding referred to a loss of more than $200 \mathrm{~mL}$ during the operative procedure and gallbladder perforation referred to gross intraperitoneal bile contamination requiring irrigation. For postoperative complications, intraabdominal hemorrhage referred to bleeding requiring transfusion, radiological, or surgical intervention; bile leak referred to persistent bile drainage from the drain site up to the 7th postoperative day or leakage requiring intervention; and voiding difficulty referred to the ongoing requirement for urinary catheterization up to the 7th postoperative day.

\section{Statistical analysis}

Data were described as means \pm standard deviations, or as medians and ranges. Continuous variables were compared using the independent $t$-test, while categorical variables were compared using the chi-squared test. Multiple regression analyses were performed using a proportional hazards model to identify factors independently associated with the LOS greater than the 80th percentile after cholecystectomy, and to estimate corresponding odds ratio (OR) in $95 \%$ confidence intervals (CI). Statistical analysis was performed using SPSS version 15.0 (SPSS Inc., Chicago, IL). Statistical significance was accepted for $P$-values less than 0.05 .

\section{Results}

Determining the cut-off value of the NLR and comparison of preoperative variables

A total of 632 patients who underwent cholecystectomy owing to symptomatic cholecystitis during the study 
period were included in this study. The median age was 55.2 (13 - 91) years, and the patients comprised 335 women (53.0\%) and 297 men (47.0\%). Of these, 511 patients (80.9\%) exhibited calculous cholecystitis and 121 patients (19.1\%) exhibited acalculous cholecystitis. Pathologic examination confirmed the presence of simple cholecystitis in 503 patients (79.6\%) and severe cholecystitis in 129 patients (20.4\%). Regarding the admission route, 283 patients $(44.3 \%)$ were admitted via the emergency department (ED) and 352 (55.7\%) were admitted via the outpatient clinic.

An ROC curve was established to determine the cutoff value for preoperative NLR that could discriminate between simple cholecystitis and severe cholecystitis. The ROC area under the curve was 0.775 . With an NLR value of 3.090 , the sensitivity and specificity were $70.5 \%$ and $70.0 \%$, respectively (Figure 1 ). Therefore, we considered 3.0 as the cutoff value, and divided the patient population into two groups: those with preoperative NLR values below $3.0(\mathrm{n}=376)$ and those with values equal to or greater than $3.0(\mathrm{n}=256)$.

When comparing preoperative variables, the two groups showed differences in age, sex, severity of cholecystitis, serum leukocyte count, presenting symptoms, and admission route; the higher NLR (NLR $\geq 3$ ) group included more patients who had advanced age $(\mathrm{p}=0.001)$, were male ( $p=0.001)$, had severe cholecystitis $(\mathrm{p}<0.001)$, had higher leukocyte count $(\mathrm{p}<0.001)$, and had initially visited the ED ( $\mathrm{p}<0.001)$ (Table 1). Both groups did not show any differences in other variables, such as the body mass index and gallbladder contents.

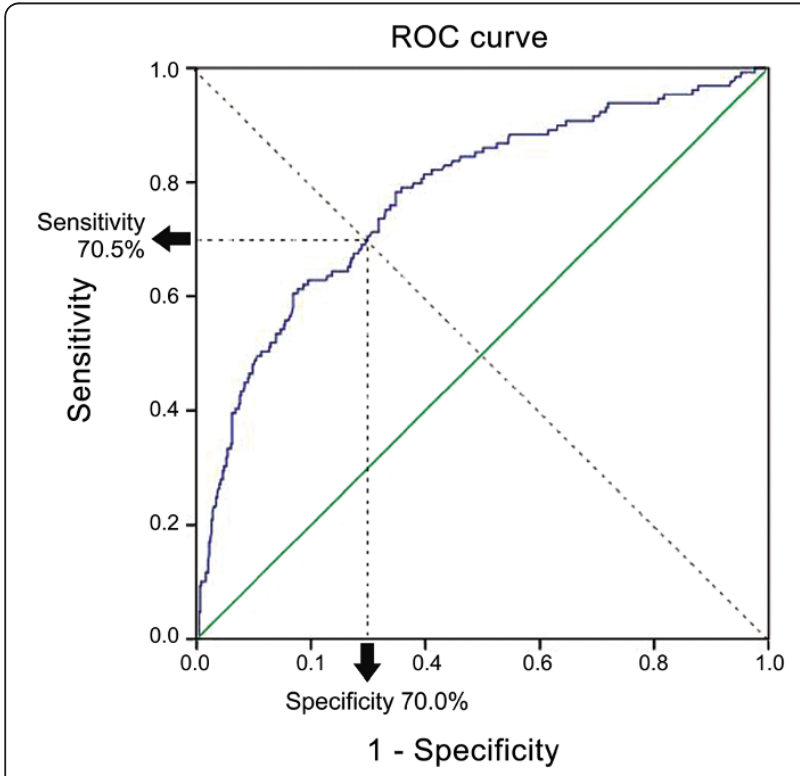

Figure 1 Receiver operating characteristic (ROC) curve for simple and severe cholecystitis. The area under ROC curve: 0.775. 95\% Cl 0.727 - 0.822; p-value <0.001.

\section{Comparison of operative and postoperative variables}

Next, surgical outcomes were compared between the groups (Table 2). Cholecystectomies were performed mainly laparoscopically, using between one and four ports. The two groups showed no difference in the operative approach and conversion rates. However, the higher NLR group had a longer operation time (median values; 77.5 min vs. $65.0 \mathrm{~min}, \mathrm{p}<0.001$ ) and longer LOS (median values; 5.0 days vs. 3.0 days, $\mathrm{p}<0.001$ ). In addition, Jackson-Pratt drains were more commonly placed in the higher NLR group $(48.4 \%$ vs. $35.9 \%$, $\mathrm{p}=0.002)$. The higher NLR group also showed higher incidence of postoperative complications, but it was not significant ( $4.3 \%$ vs. $8.2 \%, \mathrm{p}=0.056)$.

\section{Analysis of factors affecting the LOS}

Overall, the median LOS was 4.0 days (range, $1-24$ days), and the 80th percentile of LOS was 6 days. We then divided patients as either control (LOS $<6$ days) or those with prolonged LOS ( $\geq 6$ days) (Table 3 ). Univariate and multivariate analyses were conducted to identify the factors associated with prolonged LOS. On univariate analysis, patient age $(\mathrm{p}<0.001)$, male gender $(\mathrm{p}=0.036)$, preoperative leukocyte count $(\mathrm{p}=0.001)$, preoperative NLR ( $p<0.001)$, and admission via the ED ( $p<0.001)$ were all associated with prolonged LOS. A subsequent multivariate analysis identified that patient age $\geq 50$ years (OR 2.321, 95\%CI 1.47-3.630, $\mathrm{p}<0.001$ ), preoperative NLR $\geq 3.0$ (OR 1.876, 95\% CI 1.246-2.825, p =0.003), and admission via the ED (OR 1.764, 95\% CI 1.170-2.660, p =0.007) were independent factors associated with prolonged LOS.

\section{Discussion}

Since severe cholecystitis is associated with more adverse clinical features than simple cholecystitis, prompt detection of the severe cholecystitis and surgical intervention before its further advancement is essential to avoid complications related to advanced histology. Patients with severe cholecystitis are at an increased risk of damage to the main biliary ducts, ligation of aberrant hepatic ducts, and injury to the right hepatic artery during surgery [10]. Thus far, the detection of severe cholecystitis has typically relied on imaging studies, such as abdominal ultrasound and computerized tomography (CT) scanning. However, these studies occasionally fail to detect severe cholecystitis [2,5]. In the present study, we demonstrated that a preoperative NLR of 3.0 has the potential to differentiate between simple and severe cholecystitis. Therefore, the NLR calculation could be used to determine surgical priority by improving diagnostic accuracy when the CT findings are ambiguous, and by predicting a patient's risk of progressing from simple to severe cholecystitis. 
Table 1 Demographic and preoperative characteristics of patients who underwent cholecystectomy due to cholecystits grouped by NLR

\begin{tabular}{|c|c|c|c|c|}
\hline & $\begin{array}{l}\text { Total patients } \\
(\mathrm{n}=632)\end{array}$ & $\begin{array}{l}\text { NLR <3 } \\
(n=376)\end{array}$ & $\begin{array}{l}\text { NLR } \geq 3 \\
(n=256)\end{array}$ & p-value \\
\hline Age, years & $55.18(13-91)$ & $53.48(17-91)$ & $57.68(13-89)$ & 0.001 \\
\hline Sex & & & & 0.001 \\
\hline Men & $297(47.0)$ & $156(41.5)$ & $141(55.1)$ & \\
\hline Women & $335(53.0)$ & $220(58.5)$ & $115(44.9)$ & \\
\hline Body-mass index & $24.51 \pm 3.81$ & $24.59 \pm 3.86$ & $24.35 \pm 3.72$ & 0.445 \\
\hline Previous abdominal surgery (\%) & $140(22.2)$ & $89(23.7)$ & $51(19.9)$ & 0.284 \\
\hline Severity of cholecystitis & & & & $<0.001$ \\
\hline Simple cholecystitis & $503(79.6)$ & $342(91.0)$ & $161(62.9)$ & \\
\hline Severe cholecystitis* & $129(20.4)$ & $34(9.0)$ & $95(37.1)$ & \\
\hline Content in the gallbladder & & & & 0.122 \\
\hline No & $121(19.1)$ & $64(17.0)$ & $57(22.3)$ & \\
\hline Stone(s) & $511(80.9)$ & $312(83.0)$ & $199(77.7)$ & \\
\hline \multirow[t]{2}{*}{ Serum leukocyte count } & 7700 & 6200 & 11300 & $<0.001$ \\
\hline & $(1500-39300)$ & $(2700-16500)$ & $(1500-39300)$ & \\
\hline Presence of symptom & & & & 0.038 \\
\hline Abdominal pain & $586(92.7)$ & $342(91.0)$ & $244(95.3)$ & \\
\hline Indigestion (discomfort) & $25(4.0)$ & $19(5.1)$ & $6(2.3)$ & \\
\hline Fever/Chill & $7(1.1)$ & $3(0.8)$ & $4(1.6)$ & \\
\hline Others & $14(2.2)$ & $12(3.2)$ & $2(0.8)$ & \\
\hline Admission route & & & & $<0.001$ \\
\hline ED & $280(44.3)$ & $120(31.9)$ & $160(62.5)$ & \\
\hline Outpatient clinic & $352(55.7)$ & $256(68.1)$ & 96 (37.5) & \\
\hline Time to incision & & & & 0.497 \\
\hline Mean $\pm S D$ & $35.1 \pm 27.0$ & $34.5 \pm 26.9$ & $36.0 \pm 27.2$ & \\
\hline Median (range) & $23.0(1-120)$ & $22.0(1-120)$ & $24.0(2-119)$ & \\
\hline
\end{tabular}

Abbreviations: ED Emergency department, NLR neutrophil-to-lymphocyte ratio, SD standard deviation.

*Severe cholecystitis included emphysematous cholecystitis, gangrenous cholecystitis, xanthogranulomatous cholecystitis, and perforated cholecystitis.

Patients with advanced inflammatory or malignant diseases usually present with elevated NLR as a manifestation of the systemic inflammatory response. Many investigators have attempted to identify the association between the NLR and its underlying molecular basis, and have found that there is an elevation in the levels of pro-inflammatory cytokines (e.g., IL-1ra, IL-6, IL-7, IL-8, IL-12) in the plasma of patients with elevated NLR [11-13]. These inflammatory cytokines are expected to perpetuate a tissue microenvironment favoring aggressive inflammation or tumoral behavior. Furthermore, high peritumoral infiltration of macrophages was observed in cancer patients with elevated NLR [12]. Therefore, elevated NLR appears to be an accurate indicator of up-regulation of the innate immune response.

The representative forms of severe cholecystitis are gangrenous cholecystitis and gallbladder perforation. Gangrenous cholecystitis occurs in up to $30 \%$ of patients with cholecystitis [5], wherein inflammation causes interruption of blood flow to the gallbladder, resulting in gangrenous change. The mortality rate was reported to be up to $22 \%$, and it is directly related to other severe complications, such as gallbladder perforation, abscess formation, and peritonitis [14]. Gallbladder perforation is the eventual result of severe cholecystitis, where inflammation can either be localized or spread throughout the whole abdominal cavity via the perforated gallbladder [15]. In both forms, inflammation is expected to result in elevated NLR, which validates our results.

This study shows the usefulness of preoperative NLR in predicting prognosis and therefore, in determining operative priority in patients with cholecystitis. Patients with acute severe cholecystitis have higher incidences of postoperative complications and a prolonged LOS [16-19]. In this study, high NLR was found to be a predictor of severe cholecystitis as well as an independent risk factor for 
Table 2 Intra-operative and postoperative characteristics of patients who underwent cholecystectomy due to cholecystits grouped by NLR

\begin{tabular}{|c|c|c|c|c|}
\hline & $\begin{array}{l}\text { Total } \\
(n=632)\end{array}$ & $\begin{array}{l}\text { NLR <3 } \\
(n=376)\end{array}$ & $\begin{array}{l}\text { NLR } \geq 3 \\
(n=256)\end{array}$ & p-value \\
\hline Operative procedure & & & & 0.833 \\
\hline Open cholecystectomy & $6(0.9)$ & $1(0.3)$ & $5(2.0)$ & \\
\hline \multicolumn{5}{|l|}{ Laparoscopic cholecystectomy } \\
\hline - 4 port procedure & $204(32.3)$ & $120(31.9)$ & $84(32.8)$ & \\
\hline - 3 port procedure & $66(10.4)$ & $43(11.4)$ & $23(9.0)$ & \\
\hline - 2 port procedure & $307(48.6)$ & $174(46.3)$ & $133(52.0)$ & \\
\hline - single port procedure & $49(7.8)$ & $38(10.1)$ & $11(4.3)$ & \\
\hline Mean operative time (min) & & & & $<0.001$ \\
\hline Mean \pm SD & $76.5 \pm 32.7$ & $70.3 \pm 25.4$ & $85.6 \pm 39.5$ & \\
\hline Median (range) & $70.0(30-370)$ & $65.0(30-175)$ & $77.5(30-370)$ & \\
\hline Insertion of a drain & & & & 0.002 \\
\hline No & $373(59.0)$ & $241(64.1)$ & $132(51.6)$ & \\
\hline Yes & $259(41.0)$ & $135(35.9)$ & $124(48.4)$ & \\
\hline Open conversion & $4(0.6)$ & $2(0.5)$ & $2(0.8)$ & 0.485 \\
\hline Addition of one or more port(s) & $5(0.8)$ & $4(1.1)$ & $1(0.4)$ & \\
\hline Frequency of total analgesics & & & & 0.385 \\
\hline Mean \pm SD & $3.3 \pm 3.9$ & $3.2 \pm 3.7$ & $3.5 \pm 4.3$ & \\
\hline Median (range) & $2.0(0-22)$ & $2.0(0-20)$ & $2.0(0-22)$ & \\
\hline Postoperative hospital stay (days) & & & & $<0.001$ \\
\hline Mean \pm SD & $4.4 \pm 2.8$ & $3.8 \pm 2.1$ & $5.2 \pm 3.4$ & \\
\hline Median (range) & $4.0(1-24)$ & $3.0(1-23)$ & $5.0(1-24)$ & \\
\hline Postoperative complications & $37(5.9)$ & $16(4.3)$ & $21(8.2)$ & 0.056 \\
\hline Voiding difficulty & 8 & 4 & 4 & \\
\hline Pleural effusion & 8 & 4 & 4 & \\
\hline Wound infection & 5 & 2 & 3 & \\
\hline Pneumonia & 5 & 2 & 3 & \\
\hline Bleeding & 4 & 2 & 2 & \\
\hline Bile leakage & 3 & 1 & 2 & \\
\hline Pneumothorax & 1 & 1 & 0 & \\
\hline Pancreatitis & 1 & 0 & 1 & \\
\hline Delayed gastric emptying & 2 & 0 & 2 & \\
\hline
\end{tabular}

Abbreviations: NLR neutrophil-to-lymphocyte ratio, SD standard deviation.

prolonged LOS. Early cholecystectomy was shown to decrease LOS in patients with acute severe cholecystitis [20]. Therefore, prioritizing patients with high NLR for operation would reduce postoperative morbidity and LOS. Similarly, operation time was longer in the high NLR group than in the low NLR group. However, the incidences of open conversion or the addition of one or more port(s) were similar in the two groups.

To our knowledge, this study is the first to investigate the relationship between the NLR and cholecystitis. According to the disease entities or their severity, a range of NLR cut-off values have been proposed, usually from
3 to 8 [21]. Of these, a threshold of $>5.0$ has been most frequently proposed [22-26], while recent reports have recommended a value of 3.0 [27-31]. We determined the cut-off value of severe cholecystitis as 3.0 based on our ROC curve analysis; the NLR value of 3.0 had an acceptable reliability in the analysis $(70.5 \%$ sensitivity and a specificity of $70.0 \%)$. Therefore, we believe that a NLR cut-off value of 3.0 is suitable, and consistent with previous studies [27-31]. However, further study is needed to validate our cut-off value, and to more precisely identify an optimal NLR with the greatest prognostic power in cholecystitis. 
Table 3 Odds ratio for increased length of hospital stay ( $\geq 6$ days; 80percentile) associated with clinical variables in patients undergoing cholecystectomy due to cholecystitis

\begin{tabular}{|c|c|c|c|c|c|c|}
\hline & \multicolumn{3}{|c|}{ Univariate analysis } & \multicolumn{3}{|c|}{ Multivariate analysis } \\
\hline & Odds ratio & 95\% C.I. & p-value & Odds ratio & 95\% C.I. & p-value \\
\hline Age & & & $<0.001$ & & & $<0.001$ \\
\hline \multicolumn{7}{|l|}{$<50$ years (standard) } \\
\hline$\geq 50$ years & 2.553 & $1.642-3.970$ & & 2.312 & $1.472-3.630$ & \\
\hline Sex & & & 0.036 & & & \\
\hline \multicolumn{7}{|l|}{ Woman (standard) } \\
\hline Man & 1.508 & $1.036-2.195$ & & & & \\
\hline Body-mass index & & & 1.000 & & & \\
\hline \multicolumn{7}{|l|}{$<25$ (standard) } \\
\hline$\geq 25$ & 0.988 & $0.677-1.440$ & & & & \\
\hline History of prior laparotomy & & & 0.491 & & & \\
\hline \multicolumn{7}{|l|}{ No (standard) } \\
\hline Yes & 0.829 & $0.521-1.318$ & & & & \\
\hline Preoperative leukocyte count $\left(/ \mathrm{mm}^{3}\right)$ & & & 0.001 & & & \\
\hline \multicolumn{7}{|l|}{$<10,000$ (standard) } \\
\hline$\geq 10,000$ & 1.928 & $1.307-2.844$ & & & & \\
\hline Preoperative NLR & & & $<0.001$ & & & 0.003 \\
\hline \multicolumn{7}{|l|}{$<3.0$ (standard) } \\
\hline$\geq 3.0$ & 2.482 & $1.696-3.633$ & & 1.876 & $1.246-2.825$ & \\
\hline Admission via ED & & & $<0.001$ & & & 0.007 \\
\hline \multicolumn{7}{|l|}{ No (standard) } \\
\hline Yes & 2.172 & $1.485-3.178$ & & 1.764 & $1.170-2.660$ & \\
\hline Approach of operative method ${ }^{*}$ & & & 0.613 & & & \\
\hline \multicolumn{7}{|l|}{ Conventional surgery } \\
\hline Reduced port surgery & 0.892 & $0.602-1.321$ & & & & \\
\hline
\end{tabular}

Abbreviations: ED emergency department, NLR neutrophil-to-lymphocyte ratio.

${ }^{*}$ Conventional surgery included open surgery, 3-port and 4-port cholecystectomies, and reduced port surgery includes 2-port and single-port laparoscopic cholecystectomies.

The limitations of this study are those common to all database research. Since it involved a retrospective review of prospectively collective data, the results should be confirmed by prospective trials. Moreover, when we divided our patient population according to the NLR cut-off value, patient distribution was not well-balanced; our higher NLR patients included more patients with older age and men. Therefore, age and sex may act as confounding factors, which may affect the conclusion that an NLR of 3.0 is independently related with the severity of cholecystitis. In addition, the incidence of acalculous cholecystitis herein was $19.1 \%$ which did not fall within the general range of $2 \%$ to $15 \%$ as noted by others $[32,33]$. Acute acalculous cholecystitis has been generally shown poor prognosis than acute calculous cholecystitis; the median NLRs of calculous cholecystitis and acalculous cholecystitis were 2.67 and 2.18, respectively
$(\mathrm{P}=0.004)$. Therefore, in understanding our results, the differences in the composition of patient population should be taken into consideration.

\section{Conclusions}

Routine preoperative NLR calculation in patients with cholecystitis did not only provide a simple means of identifying patients with severe cholecystitis, but also served as a surrogate marker for predicting prolonged LOS. We found that the patients with cholecystitis could be divided into low risk for severe cholecystitis (NLR $<3.0$ ) and high risk for severe cholecystitis (NLR $\geq 3.0$ ) according to the NLR value at admission. Such approach of determining the operative priority based on the NLR value is expected to induce favorable surgical outcome by satisfying the "sickest first" principle and enabling expectant perioperative management 


\section{Abbreviations}

Cl: Confidential interval; LOS: Length of hospital stay; NLR: Neutrophil-tolymphocyte ratio; OR: Odds ratio; ROC: Receiver operating characteristic

\section{Competing interests}

The authors declare that they have no competing interests.

\section{Authors' contributions}

SJK designed the study, and finally approved the version to be published, SCL and JWP equally contributed to acquiring and analyzing data, and SKL wrote the paper. All authors read and approved the final manuscript.

\section{Acknowledgements}

This study did not involve any funding body.

Received: 7 August 2014 Accepted: 11 November 2014

Published: 27 November 2014

\section{References}

1. Russo MW, Wei JT, Thiny MT, Gangarosa LM, Brown A, Ringel Y, Shaheen NJ, Sandler RS: Digestive and liver diseases statistics, 2004. Gastroenterology 2004, 126(5):1448-1453.

2. Charalel RA, Jeffrey RB, Shin LK: Complicated cholecystitis: the complementary roles of sonography and computed tomography. Ultrasound Q 2011, 27(3):161-170.

3. Ahmad MM, Macon WL: Gangrene of the gallbladder. Am Surg 1983, 49(3):155-158

4. Wilson AK, Kozol RA, Salwen WA, Manov LJ, Tennenberg SD: Gangrenous cholecystitis in an urban VA hospital. J Surg Res 1994, 56(5):402-404.

5. Bennett GL, Rusinek H, Lisi V, Israel GM, Krinsky GA, Slywotzky CM, Megibow A CT findings in acute gangrenous cholecystitis. AJR Am J Roentgenol 2002, 178(2):275-281.

6. Morfin E, Ponka JL, Brush BE: Gangrenous cholecystitis. Arch Surg 1968, 96(4):567-573.

7. Kwon HC, Kim SH, Oh SY, Lee S, Lee JH, Choi HJ, Park KJ, Roh MS, Kim SG, Kim HJ, Lee JH: Clinical significance of preoperative neutrophillymphocyte versus platelet-lymphocyte ratio in patients with operable colorectal cancer. Biomarkers: Biochem Indicat Expo Response Susceptibility Chem 2012, 17(3):216-222

8. Proctor MJ, Morrison DS, Talwar D, Balmer SM, Fletcher CD, O'Reilly DS, Foulis AK, Horgan PG, McMillan DC: A comparison of inflammation-based prognostic scores in patients with cancer. A Glasgow Inflammation Outcome Study. Eur J Cancer 2011, 47(17):2633-2641.

9. Tamhane UU, Aneja S, Montgomery D, Rogers EK, Eagle KA, Gurm HS: Association between admission neutrophil to lymphocyte ratio and outcomes in patients with acute coronary syndrome. Am J Cardiol 2008, 102(6):653-657.

10. Davis B, Castaneda G, Lopez J: Subtotal cholecystectomy versus total cholecystectomy in complicated cholecystitis. Am Surg 2012, 78(7):814-817.

11. Kantola T, Klintrup K, Vayrynen JP, Vornanen J, Bloigu R, Karhu T, Herzig KH, Napankangas J, Makela J, Karttunen TJ, Tuomisto A, Makinen MJ: Stagedependent alterations of the serum cytokine pattern in colorectal carcinoma. Br J Cancer 2012, 107(10):1729-1736.

12. Motomura T, Shirabe $K$, Mano $Y$, Muto J , Toshima T, Umemoto Y, Fukuhara T, Uchiyama H, Ikegami T, Yoshizumi T, Soejima Y, Maehara Y: Neutrophillymphocyte ratio reflects hepatocellular carcinoma recurrence after liver transplantation via inflammatory microenvironment. J Hepatol 2013, 58(1):58-64

13. Ishizuka M, Nagata H, Takagi K, Iwasaki Y, Kubota K: Combination of platelet count and neutrophil to lymphocyte ratio is a useful predictor of postoperative survival in patients with colorectal cancer. $\mathrm{Br} J$ Cancer 2013, 109(2):401-407.

14. Grant $\mathrm{RL}$, Tie ML: False negative biliary scintigraphy in gangrenous cholecystitis. Australas Radiol 2002, 46(1):73-75.

15. Derici H, Kara C, Bozdag AD, Nazli O, Tansug T, Akca E: Diagnosis and treatment of gallbladder perforation. World J Gastroenterol: WJG 2006, 12(48):7832-7836.

16. Bingener J, Stefanidis D, Richards ML, Schwesinger WH, Sirinek KR: Early conversion for gangrenous cholecystitis: impact on outcome. Surg Endosc 2005, 19(8):1139-1141.
17. Choi SB, Han HJ, Kim CY, Kim WB, Song TJ, Suh SO, Kim YC, Choi SY: Early laparoscopic cholecystectomy is the appropriate management for acute gangrenous cholecystitis. Am Surg 2011, 77(4):401-406.

18. Falor AE, Zobel M, Kaji A, Neville A, De Virgilio C: Admission variables predictive of gangrenous cholecystitis. Am Surg 2012, 78(10):1075-1078.

19. Tsushimi T, Matsui N, Takemoto Y, Kurazumi H, Oka K, Seyama A, Morita T: Early laparoscopic cholecystectomy for acute gangrenous cholecystitis. Surg Laparosc Endosc Percutan Tech 2007, 17(1):14-18.

20. Murata A, Okamoto K, Matsuda S, Kuwabara K, Ichimiya Y, Matsuda Y, Kubo T, Fujino Y: Multivariate analysis of factors influencing length of hospitalization and medical costs of cholecystectomy for acute cholecystitis in Japan: a national database analysis. Keio J Med 2013, 62(3):83-94.

21. Guthrie GJ, Charles KA, Roxburgh CS, Horgan PG, McMillan DC, Clarke SJ: The systemic inflammation-based neutrophil-lymphocyte ratio: experience in patients with cancer. Crit Rev Oncol Hematol 2013, 88(1):218-230.

22. Carruthers R, Tho LM, Brown J, Kakumanu S, McCartney E, McDonald AC: Systemic inflammatory response is a predictor of outcome in patients undergoing preoperative chemoradiation for locally advanced rectal cancer. Colorectal Dis: Offic J Assoc Coloproctology Great Brit Ireland 2012, 14(10):e701-e707.

23. Cedres S, Torrejon D, Martinez A, Martinez P, Navarro A, Zamora E, Mulet-Margalef N, Felip E: Neutrophil to lymphocyte ratio (NLR) as an indicator of poor prognosis in stage IV non-small cell lung cancer. Clin Translational Oncol: Offic Publ Fed Spanish Oncol Soc Nat Canc Inst Mexico 2012, 14(11):864-869.

24. Chua TC, Chong CH, Liauw W, Zhao J, Morris DL: Inflammatory markers in blood and serum tumor markers predict survival in patients with epithelial appendiceal neoplasms undergoing surgical cytoreduction and intraperitoneal chemotherapy. Ann Surg 2012, 256(2):342-349.

25. Kishi Y, Kopetz S, Chun YS, Palavecino M, Abdalla EK, Vauthey JN: Blood neutrophil-to-lymphocyte ratio predicts survival in patients with colorectal liver metastases treated with systemic chemotherapy. Ann Surg Oncol 2009, 16(3):614-622

26. Sharaiha RZ, Halazun K, Mirza F, Port JL, Lee PC, Neugut Al, Altorki NK Abrams JA: Elevated preoperative neutrophil:lymphocyte ratio as a predictor of postoperative disease recurrence in esophageal cancer. Ann Surg Oncol 2011, 18(12):3362-3369.

27. Chiang SF, Hung HY, Tang R, Changchien CR, Chen JS, You YT, Chiang JM, Lin JR: Can neutrophil-to-lymphocyte ratio predict the survival of colorectal cancer patients who have received curative surgery electively? Int J Colorectal Dis 2012, 27(10):1347-1357.

28. Malietzis G, Giacometti M, Askari A, Nachiappan S, Kennedy RH, Faiz OD, Aziz $\mathrm{O}$, Jenkins JT: A preoperative neutrophil to lymphocyte ratio of 3 predicts disease-free survival after curative elective colorectal cancer surgery. Ann Surg 2013, 260(2):287-292.

29. Jeong JH, Lim SM, Yun JY, Rhee GW, Lim JY, Cho JY, Kim YR: Comparison of two inflammation-based prognostic scores in patients with unresectable advanced gastric cancer. Oncology 2012, 83(5):292-299.

30. Keizman D, Ish-Shalom M, Huang P, Eisenberger MA, Pili R, Hammers $H$, Carducci MA: The association of pre-treatment neutrophil to lymphocyte ratio with response rate, progression free survival and overall survival of patients treated with sunitinib for metastatic renal cell carcinoma. Eur J Cancer 2012, 48(2):202-208.

31. Wang S, Zhang Z, Fang F, Gao X, Sun W, Liu H: The neutrophil/lymphocyte ratio is an independent prognostic indicator in patients with bone metastasis. Oncol Lett 2011, 2(4):735-740.

32. Fox MS, Wilk PJ, Weissmann HS, Freeman LM, Gliedman ML: Acute acalculous cholecystitis. Surg Gynecol Obstet 1984, 159(1):13-16.

33. Ryu JK, Ryu KH, Kim KH: Clinical features of acute acalculous cholecystitis. J Clin Gastroenterol 2003, 36(2):166-169.

doi:10.1186/1471-2482-14-100

Cite this article as: Lee et al:: The utility of the preoperative neutrophil-tolymphocyte ratio in predicting severe cholecystitis: a retrospective cohort study. BMC Surgery 2014 14:100. 